incision too near the free edge. But in many cases the cartilage is so small that the incision has necessarily to be made very close to the cilia. And I frankly admit that under these circumstances $I$ am not able to accomplish the complete and permanent reposition of the cilia by the above operation alone; $I$ find it necossary to add to it the intermarginal incis. ion and to fill the marginal wound with a graft of skin or mucous membrane. The graft should be of the same length and width as the wound. Retaining sutures are not necessary, but both eyes should be bandaged until the graft is well adherent in its new bed (twenty-four to forty-eight hours). I use skin grafts in preference to mucous membrane, because the normal lid margin is covered with skin, not with mucous membrane; because skin grafts are less liable to drying up and mortifying and because they give a more substantial support to the cilia. I cut the required strip from the integument behind the ear, the incisions penetrating obliquely just to the corium The graft is transported directly to the lid and adjusted to the wound.

Hairs do not grow in such skin grafts. If afterward any hairs appear in the new lid margin, it is easy to prove they are cilia left behind in the posterior portion of the lid margin by a faulty execution of the intermarginal incision.

The management of entropium briefly outlined in this paper is the result of careful and critical observations and trials carried on during the past twenty years; and I feel warranted by a large clinical experience, in stating that the operative procedures here recommended satisfy every requirement of a rational operation. They do not cause any disfiguration of the lids; they rectify the primary structural changes causing the entropium; and they relieve the malposition perfectly and permanently.

\section{HINTS ON THE HYGIENE OF TRACHOMA AND THE INFLUENCE OF ALTITUDE AND CLIMATE.}

Presented to the Section on Ophthalmology at the Forty-eighth Annual Meeting of the American Medical Association at Philadelphia, Pa., June 1-4, 1897.

BY SWAN M. BURnetT, M.D., Рн.D. WASHINGTON, D. C.

To establish efficient rules for the hygienic management of a disease it is first necessary to have definite ideas in regard to its causation, the condition of the subject and the environment most conducive to its development.

In respect to trachoma our knowledge is sadly deficient as to the majority of these factors. We have not yet arrived at the unanimous acceptance of a microbic origin of the disease. While the probabilities, in view of recent bacteriologic studies, point to the existence of a specific micro-organism as concerned in some way in the production of trachoma, the most diligent researches have failed to find one which any considerable number of investigators have agreed upon as the definite trachomatous microbe. By others it is held that the disease is essentially one of the adenoid tissue of the conjunctiva, which an inflammation of that membrane brought about by any means whatever is capable of calling up in a person predisposed to it, and who is in a condition favorable for its outbreak. In other words, a positive and well established operating course for trachoma has not yet been agreed upon. Even its contagiousness per se, which was formerly accepted as one of the characteristics of the disease, is now questioned by many. It is acknowledged that the discharge from a trachomatous eye may contain micro-organisms which, when transported to another as yet unaffected conjunctiva, will set up an inflammation there, but this inflammation is not necessarily trachomatous. Any inflammation, however, is capable of setting in motion a train of processes which will end in an outbreak of trachoma if a tendency to the disease be present, just as an attack of pneumonia will hasten the appearance of tuberculosis in one predisposed to it. This matter of predisposition as applied to trachoma is one which has received considerable attention only recently. In former times it was considered a purely contagious affection to which all individuals were liable in the same degree, and this idea is still held by some.

The facts that have been elicited from a systematic investigation of the prevalence of trachoma among different people show, however, such a disparity of susceptibility among them that we are forced to consider race as an important factor in the establishment of a predisposition to the disease. Some races, as the negro in the United States, are practically immune even under the most unfavorable hygienic surroundings, while others, as the Irish, Polish Jews, Italians, etc., are especially liable and suffer greatly when there are favorable conditions for the development of the disease. With the acceptance of the theory of a dyscrasia or predisposition as probably the essential character of the affection, our ideas in respect to its hygienic management must differ somewhat from those based on the theory of its purely contagious nature. As, however, the idea of a dyscrasia has been considered seriously only within a very recent period, our knowledge of the nature of this predisposition is as yet very limited. This is a field which at present offers the most promising results for study and investigation. It must be borne in mind that here, as in all diseases of a demonstrated or supposed microbic origin, even with the acceptance of a dyscrasia or predisposition, we have to consider not only the microorganism but also the soil in which it grows and develops. We must have soil as well as seed. A large number of pathologic micro-organisms we have always with us everywhere, and in the conjunctival sac of perfectly healthy eyes there are often to be found organisms which, if they were sufficiently numerous and the condition for their growth favorable, would in a short time set up a morbid process; all they need is a proper condition of the soil. Hitherto we have been directing our attention exclusively to the microbe, its form, habits, modes of development, etc., to the almost utter neglect of the soil on what it grows and pabulum on which it feeds. It is evident, therefore, that any system of hygienic management which aims at anything like scientific exactness and sutisfactory practical results, must embrace a study of the condition of the soil on which the micro-organism grows as well as the size and shape of the organism itself. This general proposition applies to trachoma so far as it may be demonstrated to be a microbic disease. But if it shall be found that there is no specific microbe but that it is simply a disease of the adenoid tissue of the conjunctiva, there must still be something which starts the morbid process in motion, and the conditions for its action must be propitious. It seems probable that this beginning may be an inflammation of the 
conjunctiva of almost any kind. Even an ordinary catarrhal conjunctivitis, the result of dust, smoke, bad air or other irritating influence, may suffice to prepare the ground and set the process going.

The first law, therefore, in the treatment of trachoma and in the prevention of it in those predisposed to it, is the avoidance of those causes which are likely to lead to such an inflammation. They should be kept apart from those suffering from any form of contagious ophthalmia. Over-crowding and badly ventilated quarters should be avoided, and such patients should be kept out of all forms of vitiated or irritating atmosphere. They should not follow any occupation in which there is dust or smoke. Out-of-door employments are not to be unreservedly recommended, since it is precisely in these that such conditions are likely to exist, especially as regards dust. This applies with especial force to general farming; for in field work of many descriptions, harvesting, threshing, etc., there is commonly a great deal of dust, while the ammoniac fumes from the ordinary farm stable are extremely irritating to the conjunctiva. For the same reason dry and dusty localities are to be studiously avoided.

The effect of climate has not yet been studied sufficiently to enable us to form a just estimate of its significance. In and of itself temperature seems to exert but little influence. Cold and hot countries are free and affected in nearly the same degree. Humidity and dryness also are not by themselves apparently of much importance since trachoma is found in both. The influence of climate would appear to depend more largely upon the presence or absence of dust than upon anything else. Of course the habits of the people in regard to over-crowding, etc., as influenced by climate are not without their effect in this particular.

As a rule the countries bordering on the sea are less affected, probably on account of the absence of dust, while the interior lands, subsequent to long drouth, are afflicted more seriously. Some of the most malignant cases we have in the central portion of this country are to be found among the inhabitants of the country districts of Kentucky and West Virginia. Further investigation is much needed on this point, and it is hoped that observation will in future be more specifically directed to the meteorologic conditions as influencing the development of this disease.

The same may be said of altitude, for we have found that altitude alone does not give an immunity. It is seen at the height of 10,000 feet above the sea-level in Colorado and in the mountains of the Tyrol, and I have seen it among the mountains of East Tennessee 1,000 feet. On the other hand certain countries at the sea level as Ceylon are entirely free. It would seem to be a fact, however, that other things being equal a high elevation, if not dusty, exercises a beneficial effect on the course of the disease, most probably on account of its bracing effect on the general system.

\section{TEN YEARS' EXPERIENCE WITH THE SUR- GICAL, TREATMENT OF TRACHOMA.}

Presented to the Section on Ophthalmology at the Forty-eighth Annual Meeting of the American Medical Association, held at Philadelphia, June 1-4, 1897.

BY HAROLD GIFFORD, M.D. OMAHA, NEB.

Although the idea of treating trachoma surgically is at least as old as the days of ancient Greece, it received very little attention in modern times until about 1890. To be sure, occasional writers, Galezgowski, Mandelstamm, Schneller, Hotz, Prince and others, had urged upon the profession one surgical procedure or another, but when I first began to devote myself to ophthalmology in 1882 and for eight years thereafter, surgical measures had practically no place in the treatment of trachoma in a great majority of clinics in this country and in Europe. In the years 1890-92, however, ophthalmology suffered from what amounted almost to an epidemic of surgical cures for trachoma, and cutting, brushing, burning, scraping, squeezing and scarifying were recommended with an enthusiasm born of incomplete observation and hasty generalization. Nearly all of these methods were warranted to cure the great majority of cases in from one to four weeks and the prospects opened up to the general practitioner and to the oculist with a number of old trachoma cases on his hands, were so brilliant that it is not surprising that bluestone and nitrate of silver went to a discount, while Knapp's roller forceps and other mechanical appliances rose in favor correspondingly. Those, however, who had for several years previous to this epidemic been treating trachoma by surgical methods knew that these high expectations would not be fulfilled and that a reaction was inevitable. The signs of this reaction are now apparent enough and I think it not unlikely that unless the good which can be accomplished by surgical measures be urged systematically and with moderation, in fifteen years they will have no more of a place in ophthalmology than they had ten years ago. The trouble with these methods is that while each of them can do some good, no one of them is applicable to all sorts of cases, and it is even rare that any one of them is sufficient for any given case.

I began treating my trachoma cases surgically in 1886 , and while the brilliant results which at first I thought I secured afterward, in many cases, turned out to be only moderate, I have persisted in the use of squeezing, cutting, scraping, burning and scarifying, each in its proper place, combined, nearly always, with applications of nitrate of silver and sulphate of copper, and have learned to make use of them in many cases in which, by some authorities, they are considered contra-indicated. The only cases in which I consider them, as a rule, contra-indicated are those of acute trachoma; for the great majority of these, if seen within a few days from the start, can be cured in from three to six weeks (and occasionally in much less time) by a thorough daily application of the sulphate of copper crystal to the retrotarsal folds. When at the end of the latter period there are still marked signs of infiltration, the use of expression or the exci. sion of a strip from the folds is generally indicated.

With regard to the technique of expression, I use forceps, to be described later on, going over the tissues at least three times, with gradually increasing pressure. In this way the trachomatous foci are first broken up and then thoroughly squeezed out, without so much tearing of the folds as sometimes results if the attempt is made to squeeze them out completely at once. To prevent the adhesions which otherwise often give much trouble in the after-treatment, I have of late, at the close of extensive operations, everted each lid by two stitches, which are passed through the edge of the lid and the skin of the cheek or brow. A moist dressing is then applied, the moisture being retained by gutta-percha tissue and the cotton which comes next to the everted lids being well coated with 